\title{
Anomalous water and ion dynamics in hydroxyapatite mesopores
}

\author{
Túlio Honório $^{\mathrm{a}}$, Thibault Lemaire ${ }^{\mathrm{a}}$, Devis Di Tommaso ${ }^{\mathrm{b}}$, Salah Naili ${ }^{\mathrm{a}, *}$ \\ ${ }^{a}$ Laboratoire Modélisation et Simulation Multi Echelle, MSME UMR 8208 CNRS, \\ Université Paris-Est, Créteil Cedex 94010, France \\ ${ }^{b}$ School of Biological and Chemical Sciences, Queen Mary University of London, Mile End \\ Road, London E1 4NS, UK
}

\begin{abstract}
Hydroxyapatite (HAP) is the principal phase of bones, where the presence of ions in the fluids within HAP pores is critical to important phenomena such as bone remodeling, mineralization and fossilization. Classical molecular dynamics simulations of HAP pores ranging from 2 to $120 \mathrm{~nm}$, containing pure water and aqueous solutions of $\mathrm{CaCl}_{2}$ and of $\mathrm{CaF}_{2}$, were conducted to quantify the effect of confinement and solution composition on the dynamic properties of water and ions. Diffusion coefficients were obtained from formulations adapted to diffusion processes parallel and perpendicular to the HAP walls. A change in diffusion mechanism is observed in the direction perpendicular to the HAP walls: after a transition period proportional to the pore size, the mean squared displacement (MSD) scales with the square-root of the time instead of being linear. The presence of $\mathrm{CaCl}_{2}$ and $\mathrm{CaF}_{2}$ decelerates water and ion dynamics and changes in ion concentration modify the in-plane dynamics more strongly than the outplane dynamics of ions in HAP pores.

Keywords: Hydroxyapatite; confined diffusion; classical molecular dynamics; ion self-diffusion; specific ion effects.
\end{abstract}

\footnotetext{
* Corresponding author

Email address: salah.naili@univ-paris-est.fr (Salah Naili)
} 


\section{Highlights}

- The anisotropic nature of water and ions dynamics is quantified

- The deceleration effects of confinement on electrolyte dynamics are computed

- Ion concentration affects the electrolyte dynamics on an ion specific way

- Collisions of particles on HAP walls are crucial to determine out-of-plane diffusion

\section{Introduction}

Attainting a fundamental understanding of the dynamics of water solvated

10 portant phenomena involving mineralized tissues such as bone adaptation or remodelling processes [1, 2, nutrition of osteocytes that are not directly in contact with the vascular supply [3], de- and re-mineralization processes occurring in tooth enamel and dentin in response to changes in the oral fluids [4, 5, 6], and used as a substitute material for damaged bones and teeth in orthopaedic and dental applications [9. Additionally, in situ formation of HAP is recognized as a method for the conservation of cultural heritage, including sulphated stones, gypsum stuccoes, concrete, wall paints, archaeological bones and paper [10. In nanometric pores can contribute to improving the durability and effectiveness of HAP restoration and consolidation treatments.

HAP $\left(\left[\mathrm{Ca}_{10}\left(\mathrm{PO}_{4}\right)_{6}(\mathrm{OH})_{2}\right]\right)$ is the principal phase of mineralized tissues found in mammalian bone and tooth enamel and dentin, where HAP is present in the form of thin plates with colloidal size $25-50 \mathrm{~nm}$ in the a-direction, $15-25 \mathrm{~nm}$ in the $\mathbf{b}$-direction and $2.5 \mathrm{~nm}$ in the $\mathbf{c}$-axis [11. HAP platelets are stacked along c-direction and the interlayer space is filled with body fluid, which is rich in 
ions. The thickness of the resulting pores ranges from 2 to $24 \mathrm{~nm}$ [12, which correspond to mesopores $(2-50 \mathrm{~nm})$ according to IUPAC classification.

Water and ion structuration adjacent to HAP walls is recognized to impact the biomechanical response of bones [13]. Confined fluids are well-known to behave differently from bulk fluids (e.g. Brovchenko and Oleinikova [14]), notably in what concerns transport properties [15]. Additionally, specific (or Hofmeister) ions effects, which usually manifest at concentration above 0.01 molar [16, may The confinement of electrolyte solutions in micro- or mesopores may therefore resonate into the macroscopic behavior of HAP-rich materials.

Experimental evaluation of ion effects on water dynamics within bone as well as ion dynamics per se may be challenging [17]. This aspect justifies the choice 40 of in silico strategies to quantify the dynamic properties of water molecules and charged particles in bone mesopores [17. Molecular Dynamics (MD) simulation is a particularly powerful tool to understand in detail the mechanisms of diffusion and quantify the interactions of aqueous solutions in contact with ionic surfaces [18, and unlike thermodynamic properties, self-diffusion coefficients can only be evaluated by means of a MD simulation [19.

In this context, recent MD simulations conducted by some authors of this work have provided molecular-level detail about the transport of water within HAP nanopores [20, 21, 22, 23]. Di Tommaso et al. [23] showed that solvated ions in HAP pores slows the dynamics of water molecules compared to bulk liquid by enhancing the rigidity of H-bonds networks. However, the role of confinement on the mechanism of fluid and solute diffusion is still poorly understood [24, 25, 26]. For example, confinement may induce super- and sub-diffusion regimes and in the case of nanoporous HAP this change in diffusion mechanism is still to be identified. Moreover, the self-diffusion of ions as a function of the level of confinement within HAP nanopores is also to be quantified and the studies conducted so far have not provided an estimate of the in- and out-of-plane diffusion coefficient of ions [27, 20, 21, 22, 23. Finally, because of periodic boundary conditions and finite size of the simulation box, it is imperative to 
have an estimate of these effects on the dynamics of fluid and solutes using recently proposed corrections for MD simulation in slit pores [28, 29].

In this article, we examine the self-diffusion (named simply diffusion hereafter) of water and ions confined in HAP pores ranging from 2 to $120 \mathrm{~nm}$ by means of classical molecular dynamics simulations. In particular, a numerical analysis of the finite-size effects in MD simulation has been conducted. The anisotropic nature of water and ion diffusion in HAP mesopores has been quantified in terms of the ensemble averaged Mean Squared Displacement (MSD) and analyzed according to various salinities. Diffusion coefficients were obtained by means of formulations adapted to dynamic processes parallel and perpendicular to HAP walls. The effects of confinement in affecting the diffusion mechanisms, leading to subdiffusion, have also been quantified. Aqueous solutions of $\mathrm{CaCl}_{2}$ and $\mathrm{CaF}_{2}$ were considered to determine the effect of specific ions because of their biological interest. In particular, Fluoride $\left(\mathrm{F}^{-}\right)$is known to have a fundamental physicochemical role in bone cells. The results obtained in confined conditions were confronted with those of bulk solution.

\section{Material and Methods}

\subsection{Molecular model and force field}

The atomic structure of HAP was derived from the cell parameters and crystallographic data of Sudarsanan and Young [30. The resulting unit cell was hexagonal with $\mathrm{P} 6_{3} / \mathrm{m}$ space group. This unit cell was replicated 3 times in each in-plane direction and 4 times in out-of-plane direction (Fig. 1). The resulting total number of atoms in the HAP solid layer is $N_{H A P}=1584$ atoms. HAP platelets were stacked with a surface-to-surface spacing along c-direction varying from 2 to $12 \mathrm{~nm}$. The surface of HAP considered as basal plane is $\{0001\}$, which is reported to be the dominant surface in the thermodynamically-stable 85 morphology 31 .

The space in-between HAP platelets was filled with water molecules following the simulation protocol detailed in the next section. 


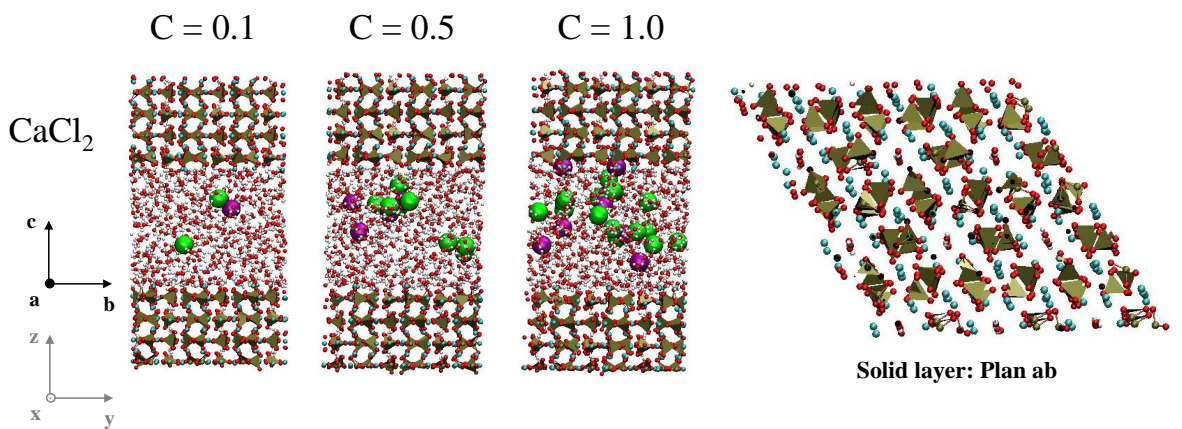

Figure 1: At left, snapshot of configuration of HAP-aqueous solution system with $H=2 \mathrm{~nm}$ at $\mathrm{CaCl}_{2}$ concentrations $C$ (in $\mathrm{mol} / \mathrm{kg}$ ) considered in this article. At right, view of the HAP solid layer from ab plan. $\mathrm{Ca}^{2+}$ and $\mathrm{Cl}^{-}$ions are depicted as purple and green van der Waals spheres, respectively. In HAP layer and water molecules, $\mathrm{O}$ atoms are red, $\mathrm{H}$ white and $\mathrm{Ca}$ cyan small spheres; P atoms are depicted as tetrahedra. For reference the orthogonal $(\mathbf{x}, \mathbf{y}, \mathbf{z})$ and crystallographic $(\mathbf{a}, \mathbf{b}, \mathbf{c})$ frames are also reported.

The interactions between the species within HAP platelet as well HAP and electrolyte were described by the force fields developed by de Leeuw and coworkers [32, 33] together with the extended single-point charge $(\mathrm{SPC} / \mathrm{E})$ water model [34].

Ions resulting from $\mathrm{CaCl}_{2}$ or $\mathrm{CaF}_{2}$ dissolution were added to the system according to four concentrations $C$ listed in Tab. 1 The dynamics of ions is reported to be concentration dependent [35, 36]. Solutions resulting from both salts have the same ionic strength $I=(1 / 2) \sum_{i} z_{i}^{2} c_{i}$, for a given concentration $c_{i}$ (in mol per unit of volume), since chloride and fluoride ions own the same valence $z_{i}$. The Debye length $1 / \kappa$ is the characteristic length associated with electrostatic interactions in colloids. This length can be directly computed from the ionic strength with $\kappa^{2}=\frac{2 I e^{2}}{\epsilon \epsilon_{0} k T}$, where $e$ is the elementary charge, $k$ is the Boltzmann constant, $T$ is the temperature, $\epsilon$ is the dieletric constant of the medium and $\epsilon_{0}$ is the vacuum permittivity. 
Table 1: Concentrations, ionic strength $I$ and Debye length $1 / \kappa$ at $310 \mathrm{~K}$ of calcium chloride and calcium fluoride.

\begin{tabular}{|c|c|c|c|}
\hline$C(\mathrm{~mol} / \mathrm{kg})$ & $M(\mathrm{~mol} / \mathrm{L})$ & $I(\mathrm{~mol} / \mathrm{L})$ & $1 / \kappa(\mathrm{nm})$ \\
\hline 0.1 & 0.104 & 0.311 & 0.926 \\
0.5 & 0.519 & 1.557 & 0.414 \\
1.0 & 1.038 & 3.114 & 0.293 \\
1.5 & 1.583 & 4.748 & 0.237 \\
\hline
\end{tabular}

\subsubsection{Molecular dynamics simulations}

MD simulations were performed using DL_POLY 4.05.1 code 37. After filling the pore with water at $1 \mathrm{~g} / \mathrm{cm}^{3}$, which is close to the density of SPC/E water at ambient conditions. The system was first equilibrated in the microcanonical (NVE) ensemble for $1 \mathrm{~ns}$. Then, a first isothermal-isobaric (NPT) run for $1 \mathrm{~ns}$ was performed to equilibrate the system under a pressure of 1 atm at $310 \mathrm{~K}$ to mimic the environment of in vivo human bone. In previous studies [20, 22, simulations in the microcanonical ensemble (NVE) were performed to ensure that the rescaling of the dynamics by the thermostat did not affect significantly the dynamics. In the production phase, the system was sampled for $2 \mathrm{~ns}$ in NPT ensemble. The Nosé-Hoover thermostat and barostat, at relaxation time $0.5 \mathrm{ps}$ for both, were employed. The long range electrostatic interactions were computed using the Smoothed Particle Mesh Ewald (SPME) method with the acceptable relative error of $10^{-6}$. This MD methodology was adopted in our previous studies of HAP nanopores [21, 22, 23].

Table 2 shows the number of water molecules $N_{w}$ for each target pore size $H$. The pore size $H$ is defined as the projection of $\mathbf{c}$ vector on $\mathbf{z}$-direction minus the effective thickness $h_{H A P}$ of the solid HAP. This effective thickness $h_{H A P}$ is defined as the distance between the center of the out-most oxygen (the out-most species in HAP surface) in each exposed HAP surface projected on z-direction plus twice the van der Waals radius of oxygen $r_{O}=0.152 \mathrm{~nm}$. Accordingly, the average pore size $\langle H\rangle_{N P T}$, sampled during the second NPT run, is computed by: $\langle H\rangle=\langle\mathbf{c} . \mathbf{z}\rangle_{N P T}-\left\langle h_{H A P}\right\rangle_{N P T}$. Similar definitions of effective thickness 
Table 2: Details of HAP-water systems (no salt), for a system with $N_{H A P}=1584$ atoms in HAP solid layer.

\begin{tabular}{|c|c|c|}
\hline$H(\mathrm{~nm})$ & $\langle H\rangle(\mathrm{nm})$ & $N_{w}$ \\
\hline 2 & 1.86 & 455 \\
3 & 2.98 & 682 \\
4 & 3.83 & 910 \\
6 & 6.11 & 1363 \\
8 & 7.97 & 1823 \\
10 & 10.09 & 2276 \\
12 & 12.16 & 2732 \\
\hline
\end{tabular}

125 and pore size were used for other layered materials such as clays [38.

\subsection{Quantification of self-diffusion coefficients under confinement}

The dynamics of fluids can be quantified in terms of the ensemble averaged (i.e. an average over the number of particles, denoted $\langle$.$\rangle hereafter) of the Mean$ Squared Displacement (MSD):

$$
\left\langle r_{i}(t)^{2}\right\rangle \approx D_{i i} t^{\alpha} \text { with } i=x, y \text { or } z
$$

${ }_{130}$ where $r_{i}$ are the components of the displacement vector $\mathbf{r}(t)=\mathbf{p}(t)-\mathbf{p}(0)$, which is function of the position $\mathbf{p}$ of the particle at time $t$. This equation holds for systems with orthogonal symmetry (see Prakash et al. 21] for a general formulation). The mechanism of diffusion can be characterised in terms of the anomalous diffusion exponent $\alpha: \alpha=1$ corresponds to Fickean diffusion and other values are associated with a subdiffusion $(0<\alpha<1)$ or superdiffusion $(\alpha>1)$ regimes. The diffusion coefficient $D_{i i}$ has dimension $\left[\right.$ length $\left.{ }^{2} .\left(\mathrm{time}^{-\alpha}\right)\right]$. In the Fickean diffusion regime it is implicitily assumed [25] (1) the independence of individual particles, (2) the existence of a sufficiently small timescale beyond which individual displacements are statistically independent, and (3) the particle displacement (during this timescale) corresponding to a typical mean free path distributed symmetrically in positive or negative directions. In anomalous 
diffusion, one or more of these assumption are violated and lead to the break of ergodicity in the behavior of the system [25]. Models such as the Continuous Time Random Walk (CTRW) [39, 40, scaled and fraction Brownian motion, and Langevin motion are often employed to describe anomalous diffusion [25].

In isotropic systems showing Fickean dynamics, the diffusion coefficient can be computed using the Einstein-Kubo equation:

$$
D_{i s o}^{E K}=\frac{1}{2 d_{n}} \lim _{t \rightarrow \infty} \frac{\left\langle|\mathbf{r}(t)|^{2}\right\rangle}{t}
$$

where $d_{n}$ is the (space) dimension of the system and the operator $\mid$. | denotes the module of a vector. According to this equation, the diffusion coefficient is the long-time limiting slope of the mean squared displacement. Therefore, a sufficiently long sampling time in MD simulations is necessary to capture such long-time limit reponse [41, 42]. However, one must also consider a sufficiently short sampling time in MD simulations to prevent dwindling statistical accuracy as the sampling time approaches the simulation duration [41].

Under confinement, the motion of particles in certain directions are not allowed leading therefore to an anomalous diffusion [24, 43, 25, 26]. For a reference orientation with the $(\mathbf{x}, \mathbf{y})$ axis parallel to the walls of a slit pore, MD simulations 21] have shown that the tensor representation of the self-diffusion tensor is diagonal. Consequently, the analysis of diffusion in HAP slit pores can be decoupled with respect to the diffusion along the wall ( $\mathbf{x}$ - and $\mathbf{y}$ combined directions) and the diffusion perpendicular (z-directions) to the walls.

\subsubsection{Diffusion parallel to the walls in a slit pore}

Given the decoupled nature of the anisotropic diffusion, parallel to the walls the diffusion coefficient can be computed using the 2D Einstein-Kubo equation:

$$
D_{\|}^{E K}=\frac{1}{4} \lim _{t \rightarrow \infty} \frac{\left\langle\left[r_{x}(t)\right]^{2}+\left[r_{y}(t)\right]^{2}\right\rangle}{t}
$$

165

Even if the diffusion along the walls remains Fickean, the dynamic of fluid can be drastically changed under nanoscale confinement due to the discrete 
nature (granularity) of matter and the interaction of fluid with the surface [28. It can be shown that the diffusion coefficient along the surface of the wall in a slit pore decreases with respect to the bulk value according to the leading order $\sigma / d$, where $\sigma$ is the diameter of the diffusing particle and $d$ is its distance to the surface [44, 45]. From the Stokes equation and after averaging over the width of the slit pore $H$, the diffusion along the wall in a slit pore with infinite lateral dimension $L \rightarrow \infty$ is given by the following expression [28]:

$$
D_{\|}^{*}(H, \infty) \approx D_{b u l k}\left[1+\frac{9}{16} \frac{\sigma}{H} \ln \left(\frac{\sigma}{H}\right)\right]
$$

where $D_{b u l k}$ is the diffusion coefficient in a bulk fluid. This reduction in the diffusion coefficient have been associated with the suppression of long-wavelength modes due to confinement [46. In this work, this expression has been used to determine the effect of confinement.

The dynamics of fluids and solutes in MD simulations are affected by the periodic boundary conditions (PBC). The origin of this artifact is due to the viscous couplings of the atoms with their periodic images, which reduces the dynamics [28, 47]. In a cubic supercell of lenght $L$, the diffusion coefficient $D_{\|}(\infty)$ (with $L \rightarrow \infty$ ) can be obtained from the diffusion coefficient $D_{\|}^{P B C}$ computed from a MD simulation with periodic boundary conditions using the following expression [48, 49, 50, 51]:

$$
D_{\|}(\infty)=D_{\|}^{P B C}+\frac{k T \xi}{6 \pi \eta L}(\text { bulk (isotropic) })
$$

where $\xi \approx 2.837298$ is the self-term for a cubic lattice at the room temperature, and $\eta$ is the viscosity of the fluid. It is noteworthy that the computations of the viscosity of $\mathrm{SPC} / \mathrm{E}$ water were reported to be insensitive to simulation cell size [49]. SPC/E water viscosity at $310 \mathrm{~K}\left(\eta=5.95 \times 10^{-4}\right.$ Pa.s $)$ can be interpolated from the results of Medina et al. [52. Note that the finite-size correction (second term in Eq. 5) is temperature dependent, which introduces a temperature dependence in $D_{\|}(\infty)$ that is not necessarily identical to the temperature dependence in $D_{\|}^{P B C}[53]$. 
For anisotropic systems, new formulations have been proposed that depend on the aspect ratio $H / L$ of the simulation box. For the HAP slit pore, we have applied the corrected versions for the parallel diffusion coefficient proposed by Simonnin et al. 28]:

$$
\begin{gathered}
D_{\|}(H>L, \infty)=D_{\|}^{P B C}-\frac{k T}{\eta}\left[\frac{3}{40} \frac{H}{L^{2}}-\frac{\ln (1+\sqrt{2})}{4 \pi L}\right] \text { (elongated box) } \\
D_{\|}(H<L, \infty) \approx D_{\|}^{P B C}+\frac{1}{24} \frac{k T}{\eta} \frac{H}{L^{2}} \text { (flat box) }
\end{gathered}
$$

These expression have been used in this work to determine corrected values of the in-plane diffusion coefficients.

\subsubsection{Diffusion perpendicular to the walls in a slit pore}

The diffusion in the $\mathbf{z}$-direction (perpendicular to the wall in a slit pore) is subjected to collisions with the walls of the pore as well as the effects of the confinement potential $V(z)$. The confinement potential $V(z)$, or potential of mean force, is related to the energy along the minimum free energy path, which is the path in phase-space that most likely the system will follow [54]. It is possible to provide estimations of $V(z)$ from the density profiles in micropores [54.

At short times, the dynamic of a single particle is expected to be Fickean since the particle does not experience reflection on the confining walls or the restoring force from the confinement potential [55]. Fickean diffusion regime holds for scales of time in which $D_{\perp} t \ll H^{2}$ [55]. The transition to a regime with subdiffusion depends on the confining potential.

In order to account for the interface, the strategy adopted here is to consider only regions within a certain distance from the surface of the nanopore, where the confinement potential $V(z)$ can be considered as constant [54. The solution of the Smoluchowski equation for a constant potential $V(z)$ gives the following expression for the MSD in the direction perpendicular to the nanopore walls 
[56]:

$$
\left\langle\left[r_{z}(t)\right]^{2}\right\rangle=\frac{H^{2}}{6}-\frac{16 H^{2}}{\pi^{4}} \sum_{n=1(\text { odd })}^{\infty} \frac{1}{n^{4}} \exp \left[-D_{\perp}\left(\frac{n \pi}{H}\right)^{2} t\right]
$$

where the diffusion coefficient $D_{\perp}$ can be obtained by fitting the MSD $\left\langle\left[r_{z}(t)\right]^{2}\right\rangle$ profile computed from the MD simulation. With this expression, the diffusion mode is equivalent to free Brownian diffusion within an infinitely high square well potential [56]. The coefficient $D_{\perp}$ has the dimension of a normal diffusion coefficient (i.e. [length ${ }^{2}$. $\left(\right.$ time $\left.\left.^{-1}\right)\right]$ ) but with the phenomenology of Eq. 8 , the resulting scaling of the $\operatorname{MSD}\left\langle\left[r_{z}(t)\right]^{2}\right\rangle$ is anomalous. Indeed, at time $t=0$, the slope of the MSD profile is proportional to $D_{\perp}$, as in the Fickean case. However, the curve asymptotically approaches $H^{2} / 6$, which characterises a subdiffusion regime [56].

In this work, the out-of-plane diffusion coefficients of water and ions have been evaluated according to Eq. 8 but also applying the Einstein-Kubo Eq. 3

$$
D_{\perp}^{E K}=\frac{1}{2} \lim _{t \rightarrow \infty} \frac{\left\langle\left[r_{z}(t)\right]^{2}\right\rangle}{t}
$$

No finite-size or PBC corrections have been considered for the out-of-plane diffusion coefficients because of the lack of adequate corrections for the direction perpendicular to the wall.

\section{Results and Discussion}

\subsection{Finite size effects on the dynamics of water and ions}

The finite size effects (periodic boundary conditions and confinement) on

the dynamics of water and ions were investigated by replicating 2, 3 and 4 times (see Fig. 2) in the $\mathbf{x}$ - and $\mathbf{y}$-directions the simulation cells of the 0.1 $\mathrm{mol} / \mathrm{kg} \mathrm{CaCl}{ }_{2}(\mathrm{aq})$ and $\mathrm{CaF}_{2}(\mathrm{aq})$ solutions (see Fig. 1). The isotropic diffusion coefficients of the water molecules and ions obtained using the Einstein-Kubo formula (see Eq. 3) are reported in Supporting Information. 


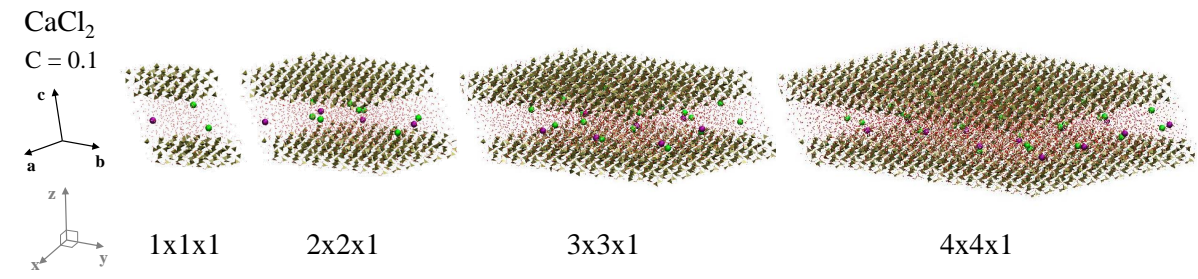

Figure 2: Snapshots of the four different simulation supercells (with $H=2 \mathrm{~nm}$ and $C=0.1$ $\mathrm{mol} / \mathrm{kg}$ ) used to study the finite size dependence of water and ion dynamics in HAP nanopores. From left to right, the systems contain 3888, 15552, 34992 and 62208 atoms, respectively.

Figure 3 displays the in- and out-of-plane diffusion coefficients of water and ions for the $\mathrm{CaCl}_{2}$ and $\mathrm{CaF}_{2}$ solutions as a function of the system size. The in-plane diffusion coefficient $D_{\|}^{E K}$ was computed from Eq. 3 using the $\mathbf{x}$ and $\mathbf{y}$ MSDs. The elongated (see Eq. 6) and flat (Eq. 7) corrections of Eq. 6 or Eq. 7 were applied to the diffusion coefficients computed from the MD simulations and the corresponding diffusion coefficients $D_{\|}(H, \infty)$ are also reported in the figure. Figure 3 shows that PBC corrections do not significantly change the values of the in-plane diffusion coefficients.

The in-plane diffusion coefficients are larger than the out-of-plane diffusion coefficients. The dynamics on both directions is affected by the confinement as the values of the in-plane and out-of-plane diffusion coefficients are significantly smaller than in the bulk.

The diffusion coefficient perpendicular to the HAP walls $D_{\perp}^{E K}$ was computed using Einstein-Kubo relation (see Eq. 9) and by fitting Kusumi et al. [56] formula to obtain $D_{\perp}$ (see Eq. 8), which takes into account the reflection of particles on the confining HAP walls. As can be seen in Fig. 4 the density of water is almost constant at distances exceeding circa $4 \AA$ from the center of the out-most atom (oxygens) in HAP surface. Given that the Lennard-Jones (LJ) diameter of oxygen in SPC/E water model is $3.55 \AA$, we can infer that after the first adsorbed water layer, the fluid within the pore can be approximated by bulk fluid (with constant density and confinement potential). In the following, 
we restricted our analysis of the diffusion coefficient perpendicular to HAP walls to the regions beyond the first hydration layer where the confinement potential $V(z)$ considered to be constant.

The dynamics of water perpendicular to $\mathrm{HAP}$ walls in the $\mathrm{CaF}_{2} 0.1 \mathrm{~mol} / \mathrm{kg}$ solutions is shown in Fig 5. Similar results were obtained for the $\mathrm{CaCl}_{2}$ solution and are reported in Supporting Information (see Fig. S3). The scaling behavior of the MSDs (see Fig. $5(\mathrm{a}))$ changes from normal diffusion $\left(\operatorname{MSD}\left\langle\left[r_{z}(t)\right]^{2}\right\rangle\right.$ scales with $t$ ) to subdiffusion $\left(\operatorname{MSD}\left\langle\left[r_{z}(t)\right]^{2}\right\rangle\right.$ scales with $\left.\sqrt{t}\right)$. The time for the transition to the subdiffusion regime depends on the pore size $H$ but in general the smaller the pore size, the smaller the transition time. The curves with dashed lines in Fig. 5 (b) have been computed from $r_{\text {trans }}^{2}=\left[\left(H-\sigma_{O w}\right) / 2\right]^{2}$, where $\sigma_{O w}$ is the LJ diameter of the oxygen in SPC/E water model. Thus, $r_{\text {trans }}$ corresponds roughly to the average distance travelled by a water molecule that is not part of the first hydration layer before being reflected on the first hydration shell of the HAP nanopore surface. Equation 8 fits quite well the MSD obtained by MD simulations, which indicates that a free Brownian diffusion within an infinitely high square well potential (i.e. the mode associated with Eq. 8) describes quite well the effects of confinement on the perpendicular diffusion of water in HAP slit pores. The time derivatives of the MSD $\left\langle\left[r_{z}(t)\right]^{2}\right\rangle$ in Fig. ${ }_{280} 5$ (c) highlight the changes in the diffusion mechanisms from normal diffusion to subdiffusion. The sudden variations in these time derivatives, especially in the case of the smaller pores (roughly three orders of magnitude for $H=2 \mathrm{~nm}$ ), show that a Fickean interpretation of the dynamics is not suitable to describe the dynamic behavior of water in the direction perpendicular to the pore walls. For comparison purposes, the diffusion coefficients $D_{\perp}$ computed using Eq. 8 have also been reported.

Figure 6 reports the MSD of the ions in the $\mathrm{CaCl}_{2}(\mathrm{aq})$ and $\mathrm{CaF}_{2}$ (aq) solutions $(C=0.1 \mathrm{~mol} / \mathrm{Kg})$ confined in the HAP pore with $H=2 \mathrm{~nm}$. In Fig. 6. (a), the MSD computed for the in-plane directions ( $\mathbf{x}$ and $\mathbf{y}$ ) show that after few hundreds of picoseconds, the ions MSD exhibits negative time derivatives, which 

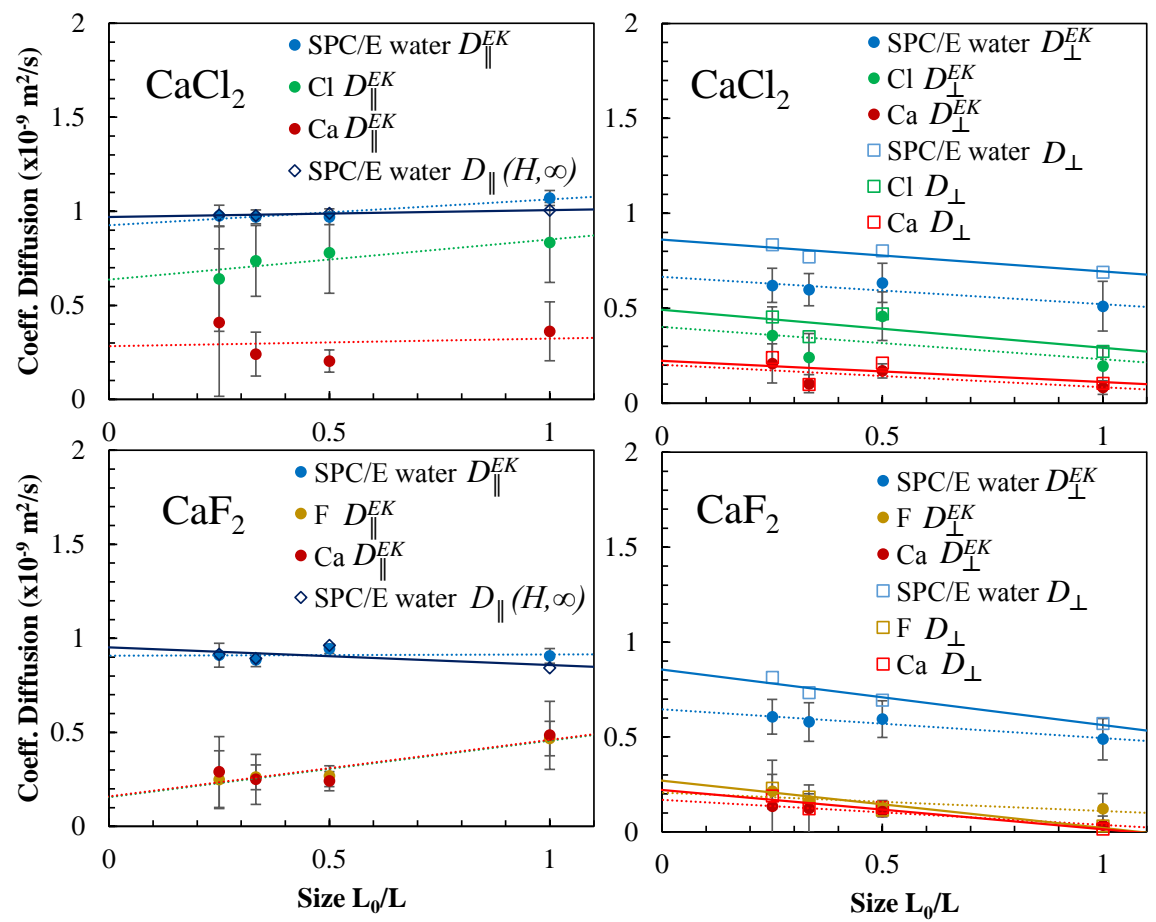

Figure 3: Diffusion coefficients of water and ions as a function of the simulation supercell (in-plane) size $L$. The quantity $L_{0}$ corresponds to the in-plane dimensions of simulation cell with $H=2 \mathrm{~nm}$ as reported in Fig. 1 and 2 (left). Full circles represent estimates of the diffusion coefficient $D_{\perp}^{E K}$ obtained using the Einstein-Kubo formula (see Eq. 3). The lines are least-square fits of the corresponding points. At right, the squares represent the estimated using Eq. 8 to obtain $D_{\perp}$, which takes into account the reflection of particle on the confining walls. 

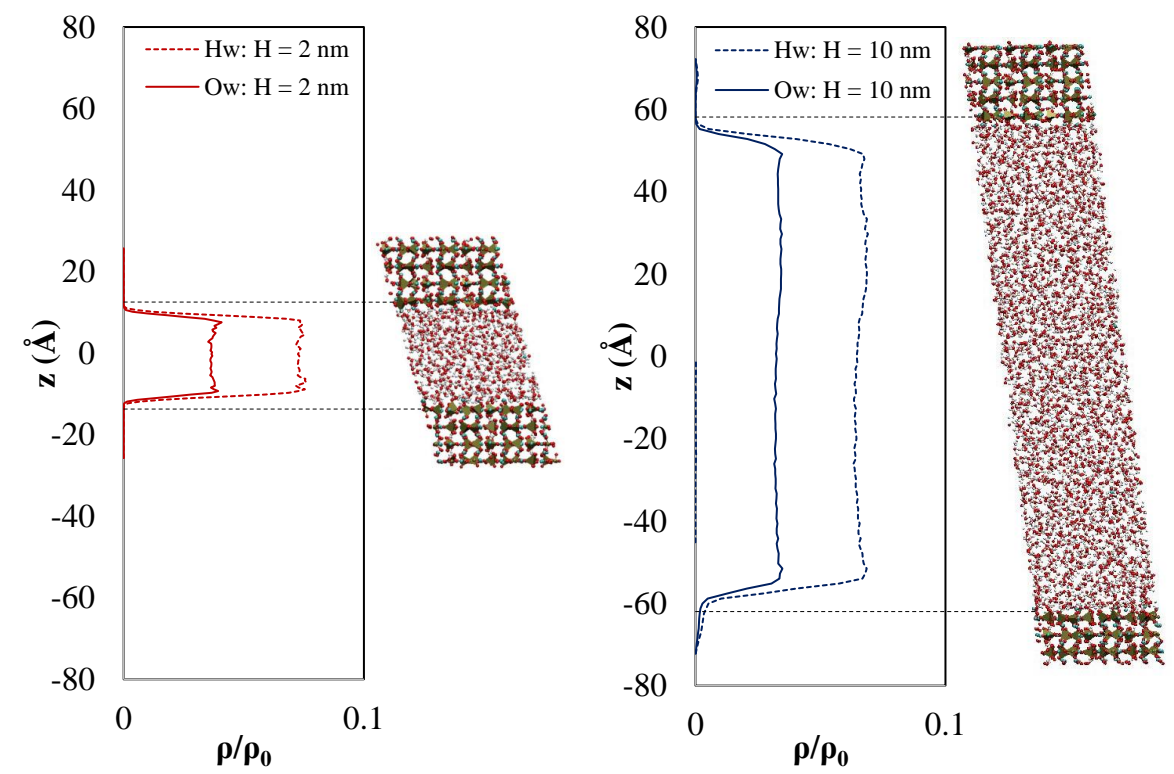

Figure 4: Density of water oxygen $\left(\mathrm{O}_{w}\right)$ and hydrogen $\left(\mathrm{H}_{w}\right)$ along $\mathbf{z}$-direction for two pores sizes $H=2 \mathrm{~nm}$ (left) and $10 \mathrm{~nm}$ (right).
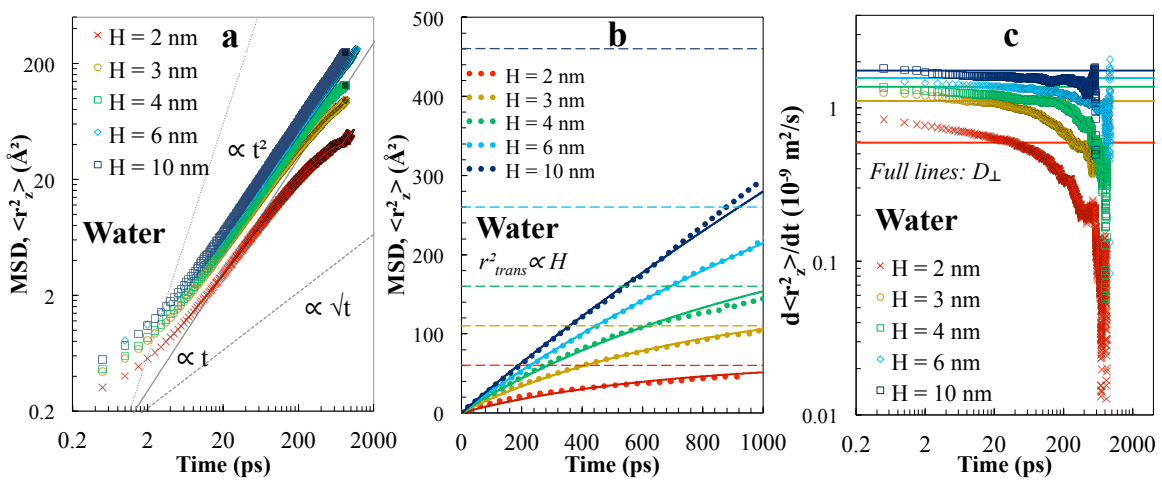

Figure 5: Dynamics of water perpendicular to HAP walls as a function of confinement: (a) log-log plot of the MSD of water (obtained from MD) for various pore sizes. The scalings with time $t$ are also shown. (b) MSD obtained from MD simulation compared to the fitting using Eq. 8 The transition to the subdiffusion regime occurs at a MSD scaling with the pore size $H$. (c) Time derivative of the MSD (symbols) and diffusion coefficient $D_{\perp}$ computed via Eq. 8 (full lines). Only $\mathrm{CaF}_{2}$ solution is considered. See the Supporting Information for results regarding $\mathrm{CaCl}_{2}$ solution. 
could be interpreted as a negative diffusion coefficient. In low concentration regimes and for the systems with the sizes considered here, only few ions are present in the simulation box, making the study of ions dynamics similar single particle tracking. In this case, the ensemble average is not enough to counteract specific random effects in the MSD of single ions. Being a stochastic process, the profile of the MSD is dependent on the amount of sampled time points [57, notably in the case of single particle diffusion. Various authors have identified the errors in the measurements of single particle dynamics (e.g. Burnecki et al. 58 ) and how they may lead to erronenous interpretations of the diffusion mechanisms [59. These uncertainties are related to the stochastic nature of diffusion and translate in the difficulty of determining if negative derivatives of the MSD are the result of systematic transport phenomena or of specific constraints associated with diffusion [60].

An interpretation of a negative diffusion of ions in salt solution could be the aggregation of the solvated ions. Nonetheless, this effect would increase with salt concentration and be more pronounced for salt with low solubility such as $\mathrm{CaF}_{2}$. However, in Fig. 6 the negative time derivative becomes less pronounced for the solutions at higher concentration and for $\mathrm{CaF}_{2}$ (aq). For larger ionic forces, there are more particles over which ensemble averages are performed and the unusual effects due to the random nature of individual particle MSD becomes less significant. Consequently, salt precipitation is unlikely to be the cause of the negative time derivatives of the MSD observed here. Indeed, even at the higher concentrations considered in this study $(C=1.0 \mathrm{~mol} / \mathrm{kg}$ ), no aggregation of ions was observed. These observations are in agreement with the hypothesis of the negative time derivatives being an artifact induced by the stochastic nature of MSD.

In single particles dynamic, corrections have been proposed accounting for the intrinsic variability of the MSD [61, 57. The black dashed line in Fig. 66 (a) corresponds to the noise-corrected MSD in the $\mathbf{x}$-direction following the approach proposed by Kepten et al. [57]. Here, the corrections with respect to both noise and ensemble heterogeneity are combined to obtain a MSD with 

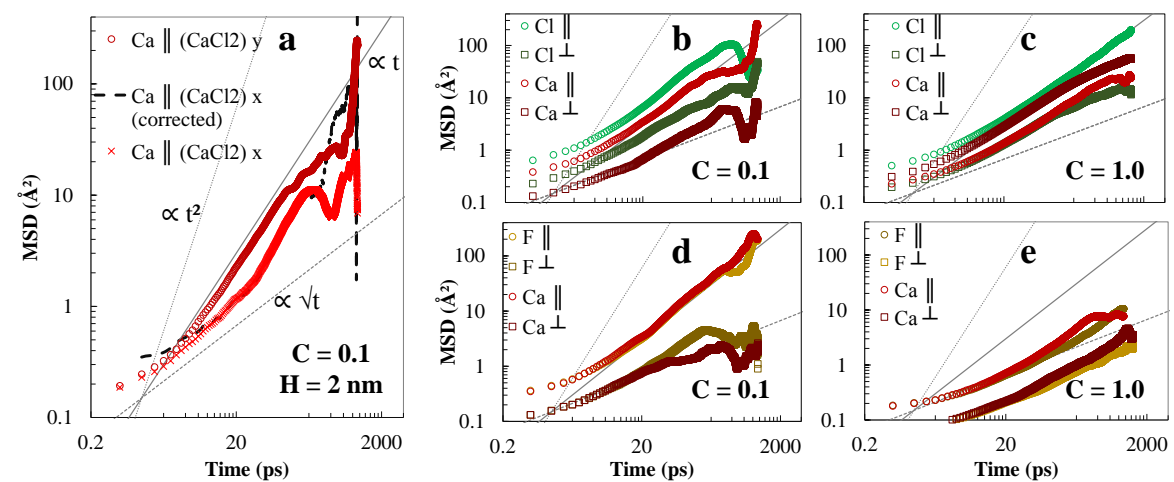

Figure 6: Dynamics of ions under confined conditions $(H=2 \mathrm{~nm})$ : (a) log-log plot of the in-plane MSD of $\mathrm{Ca}$ ions of a solution $(C=0.1)$ of $\mathrm{CaCl}_{2}$. The black dashed line corresponds to the noise-corrected MSD in x-direction following the Kepten et al. approach [57. In- and out-of-plane MSD of ions of confined solutions of $\mathrm{CaCl}_{2}$ at concentrations (b) $C=0.1$ and (c) $C=1.0$ as well as confined solutions of $\mathrm{CaF}_{2}$ at concentrations (d) $C=0.1$ and (e) $C=1.0$. In all cases, the scalings with time $t$ are shown. In all cases, all the ions in the pore were accounted for in the MSD computation.

fewer random errors. Indeed, the correction to the MSD in Fig. 6 (a) gives a MSD profile with a larger domain where time derivative remains positive.

At both low $(C=0.1)$ and higher $(C=1.0)$ salt concentrations, the MSD of ions in the direction parallel to the HAP walls scales with $t$ and consequently follows the Fickean diffusion behavior. On the other hand, at both concentrations, the MSD of ions in the direction perpendicular to the HAP walls scales approximately with $\sqrt{t}$ (subdiffusion mechanism) and this behavior is not strongly affected by salt concentration.

\subsection{Confinement and salinity effects on the dynamics of water and ions}

Figure 7 reports the in- and out-of-plane diffusion coefficients of water and ions in the $\mathrm{CaCl}_{2}$ (aq) and $\mathrm{CaF}_{2}$ (aq) solutions with concentration $C=0.1$ $\mathrm{mol} / \mathrm{kg}$ as a function of the HAP pore thickness $(2<H<12 \mathrm{~nm})$.

For in-plane diffusion, the coefficient $D_{\|}(H, \infty)$ computed via the finitesize corrected formulas (see Eq. 6] and Eq. 7) and the non-corrected diffusion coefficient $D_{\|}^{E K}$ (see Eq. 3 are compared with the analytical result of Eq. 4 . 
which provides the diffusion coefficient $D_{\|}^{*}(H, \infty)$ computed from the diffusion coefficient of each species in bulk solutions. The results are consistent with a previous study 21] showing that the in-plane diffusion coefficient of water increases with the pore size, reaching a plateau at circa $10 \mathrm{~nm}$, but this plateau is reached earlier with the corrected diffusion coefficients $D_{\|}(H, \infty)$. For the larger pores, theoretical results of $D_{\|}^{*}(H, \infty)$ (see Eq. 4) are in a reasonable agreement with the values obtained from MD simulations, whereas the reduction in the diffusion coefficient with confinement is more pronounced from MD results. For all mesopores, the in-plane diffusion coefficients of the ions are smaller than the isotropic water diffusion coefficient. Due to the limited number of ions in solution, the diffusion coefficients of $\mathrm{Ca}^{2+}, \mathrm{Cl}^{-}$and $\mathrm{F}^{-}$are more variable than the diffusion coefficient of the water molecules, but the overall trend is a slightly increase with the pore size. Calcium ions show similar in-plane diffusion coefficients irrespective of the salt concentration and counterion.

In Fig. 7 (right), the out-of-plane diffusion coefficients are shown for all the species according to the confinement. The diffusion coefficients are computed according to Einstein-Kubo relation ( $D_{\perp}^{E K}$ in Eq. 3 , assuming Fickean diffusion) as well as Kusumi et al. relation $\left(D_{\perp}\right.$ in Eq. 8, accounting for confinement and resulting subdiffusion). The Kusumi equation takes into account confinement effects in the evaluation of $D_{\perp}$ and yield slightly larger diffusion coefficients than the values obtained applying the Einstein-Kubo relation. For both in and outof-plane diffusion of water, the diffusion coefficients $D$ can be aproximated by the expression 62] $D(H) \approx D_{\text {bulk }}\left(1-e^{H / k_{d}}\right)$, where $k_{d}$ is constant obtained by fitting the values of the diffusion in the bulk fluid $D_{b u l k}$ (Supporting Information, Fig. S3).

The dynamics of water is slowed down in the presence of $\mathrm{CaF}_{2}$ when compared to $\mathrm{CaCl}_{2}$ solution. Indeed, the diffusion coefficient of chloride is larger than fluoride's, whereas calcium ions exhibit similar diffusion coefficients irre365 spective of the considered counterion. The out-of-plane diffusion coefficients of water and ions evolve non linearly with the confinement reaching a plateau at approximately $10 \mathrm{~nm}$. Such behavior is also visible for the in-plane diffusion 
coefficients.

Figure 8 shows for the nanopore with $H=2 \mathrm{~nm}$ the influence of salinity on the diffusion coefficient of water and ions as a function of the salt concentration. Three salt concentrations were considered and compared to pure water. Note that the dissolution of HAP solid walls occured at $C=1.5 \mathrm{~mol} / \mathrm{kg}$, but these simulations are not considered here. The corrections for finite-size artifacts to in-plane diffusion $D_{\|}(H, \infty)$ have been applied, and for the out-of-plane diffusion coefficients both Einstein-Kubo $D_{\perp}^{E K}$ and Kusumi et al. $D_{\perp}$ formulations were considered. The in- and out-of-plane diffusion coefficients of water decreases with the concentration of $\mathrm{CaCl}_{2}$ and $\mathrm{CaF}_{2}$. This observation is in agreement with NMR measurements or ultrafast spectroscopy results as discussed by Marcus [63]. Moreover, thsese results are consistent with previous simulation results 23. reporting that the presence of ions in HAP pores slows the dynamics of water molecules compared to bulk liquid, enhancing the rigidity of H-bonds networks. The variability of the diffusion coefficients of ions in the more dilute solutions is likely to be related to the size of the simulated systems.

Our results suggest that the in-plane diffusion coefficients of ions decrease with salt concentration. On the other hand, the concentration does not have a significant influence on the dynamics in the direction perpendicular to the HAP walls.

\section{Conclusions}

Classical MD simulations of HAP nanopores $(2 \mathrm{~nm}<H<120 \mathrm{~nm})$ in consact with aqueous electrolyte solution were conducted to obtain an atomistic description of the anisotropic dynamics of water and ions in HAP nanopores. Corrections due to PBC and finite size effects were also considered to provide confidence in the diffusion coefficients computed from MD simulations. We observed that these effects on the dynamics of water are negligible in elongated simulation boxes with the out-of-plane size not exceeding twice the average inplane size. 

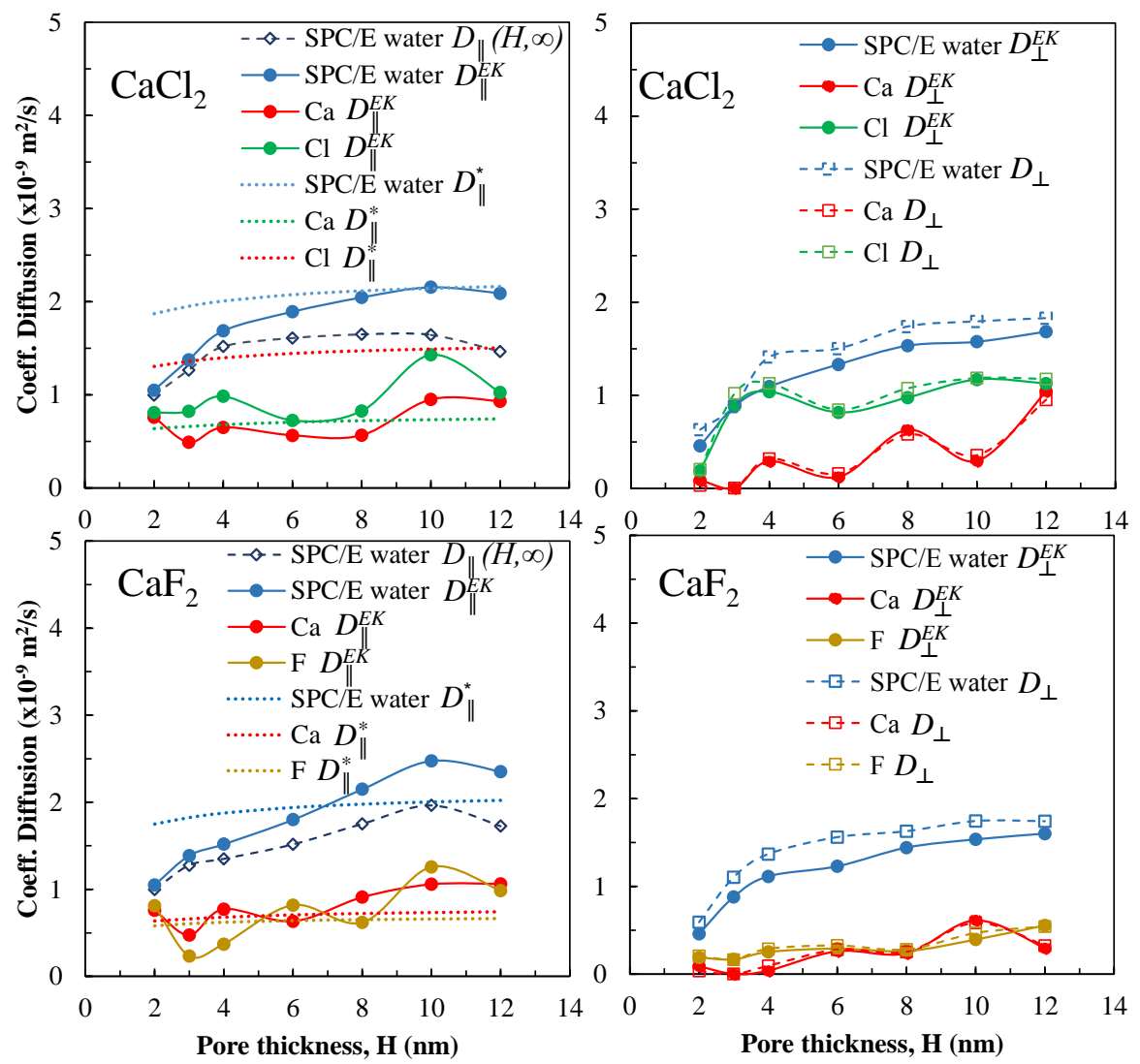

Figure 7: Diffusion coefficient of water and ions species in both in- and out-of-plane directions as a function of the pore size for a salt concentration $C=0.1 \mathrm{~mol} / \mathrm{kg}$. At left are reported the in-plane diffusion coefficients $D_{\|}(H, \infty)$ (computed via the finite-size corrected formulas Eq. 6 or Eq. 7), $D_{\|}^{E K}$ (computed with Eq. 3 and $D_{\|}^{*}(H, \infty)$ (theoretical result of Eq. 4. At right, regarding out-of-plane diffusion, the diffusion coefficient $D_{\perp}$ (computed via Kusumi et al. Eq. 8 as well as the Einstein-Kubo estimate $D_{\perp}^{E K}$ (Eq. 9 are shown. 

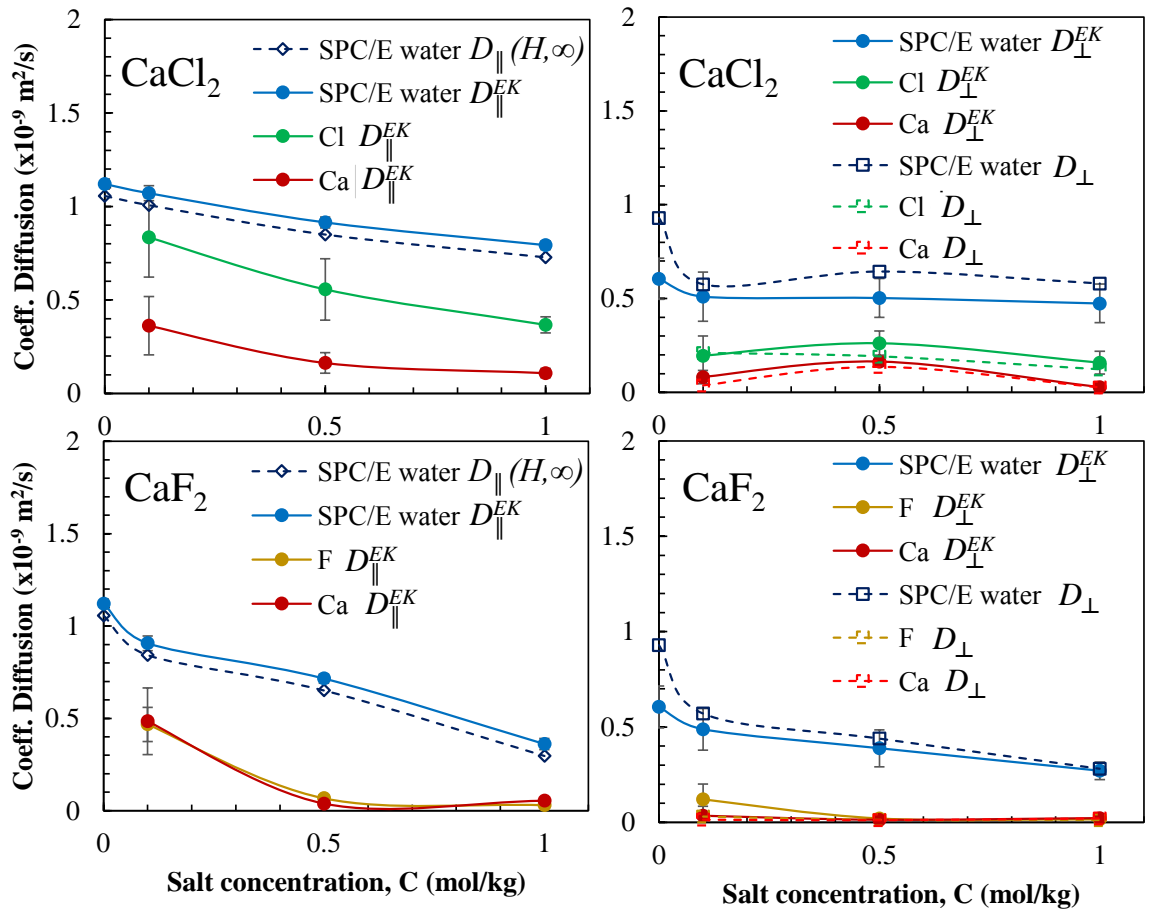

Figure 8: Diffusion coefficient of water and ions as a function of the salinity for $H=2 \mathrm{~nm}$. Diffusion coefficients defined as in Fig. 7. 
The quantification of the change in the diffusion mechanism in the direction perpendicular to HAP nanopore walls shows that after a certain transition time, proportional to the pore size, the MSD scales with $t^{\alpha}$, with $\alpha \approx 0.5$ (instead of $\alpha=1$ for a Fickean regime). This scaling is characteristic of a subdiffusion regime. We provided the diffusion coefficients of the water and ions according to the formulation of Kusumi et al. [56], which accounts for reflection of molecules on the confining walls in a mode corresponding to a free Brownian diffusion within an infinitely high square well potential. Our results suggest that this mode can describe quite well the effects of confinement on the perpendicular diffusion of water in HAP slit pores.

The variations of the diffusion coefficient with the concentration are more pronounced in the in-plane direction. Ion concentration does not show a significant influence on the dynamics in the direction perpendicular to the HAP walls. Regarding specific ion effects, the diffusion coefficient of chloride is larger that fluoride and calcium diffusion coefficients, and diffusion of calcium ions is slowed down by the presence of fluoride when compared to the presence of chloride.

Constitutive modelling of bones coping with electro-chemo-mechanical couplings can benefit of the information regarding diffusion coefficients of water and ions under confinement provided here [2]. The applications include, for instance, (i) the development of models of bone adaptation and remodeling informed by physical chemistry aspects of nanoporous HAP behavior at the molecular scale, (iii) the understanding of HAP de- and re-mineralization processes and durability according to the pore solution composition and (iii) the development of models of the ingress of aggressive agents in HAP-rich materials such as teeth and HAP treated cultural heritage objects.

\section{References}

[1] T. Lemaire and S. Naili, Medical Hypotheses 78, 367 (2012), ISSN 0306-9877, URL http://www.sciencedirect.com/science/article/ 
[2] E. Rohan, S. Naili, R. Cimrman, and T. Lemaire, Journal of the Mechanics and Physics of Solids 60, 857 (2012), ISSN 0022-5096, URL http://www. sciencedirect.com/science/article/pii/S002250961200021X.

[3] S. Mishra and M. L. Knothe Tate, The Anatomical Record Part A: Discoveries in Molecular, Cellular, and Evolutionary Biology 273A, 752 (2003), ISSN 1552-4892, URL http://onlinelibrary.wiley.com/doi/10.1002/ ar.a.10079/abstract.

[4] E. C. Moreno, M. Kresak, and D. I. Hay, Calcified Tissue International 36, 48 (1984), ISSN 0171-967X, 1432-0827, URL https://link.springer. com/article/10.1007/BF02405293

[5] G. W. Marshall, Quintessence International (Berlin, Germany: 1985) 24, 606 (1993), ISSN 0033-6572.

[6] K. Onuma, N. Kanzaki, A. Ito, and T. Tateishi, The Journal of Physical Chemistry B 102, 7833 (1998), ISSN 1520-6106, URL https://cdn-pubs. acs.org/doi/10.1021/jp981617u.

[7] R. E. M. Hedges and A. R. Millard, Journal of Archaeological Science 22, 155 (1995), ISSN 0305-4403, URL http://www.sciencedirect.com/ science/article/pii/S0305440385700179.

[8] S. W. Keenan, A. S. Engel, A. Roy, and G. Lisa Bovenkamp-Langlois, 445 - Chemical Geology 413, 18 (2015), ISSN 0009-2541, URL http://www. sciencedirect.com/science/article/pii/S0009254115300036

[9] Z. J. Shen, E. Adolfsson, M. Nygren, L. Gao, H. Kawaoka, and K. Niihara, Advanced Materials 13, 214 (2001), ISSN 1521-4095, URL a https://onlinelibrary.wiley.com/doi/abs/10.1002/1521-4095\% 450 $28200102 \% 2913 \% 3$ A3\%3C214\%3A\%3AAID-ADMA214\%3E3. 0. C0\%3B2-5.

[10] E. Sassoni, Materials 11, 557 (2018), URL http://www.mdpi.com/ $1996-1944 / 11 / 4 / 557$. 
[11] S. Weiner and W. Traub, FEBS Letters 206, 262 (1986).

[12] J. M. Holmes, D. H. Davies, W. J. Meath, and R. A. Beebe, Biochemistry 3, 2019 (1964), ISSN 0006-2960, URL http://dx.doi.org/10.1021/ bi00900a042.

[13] E. E. Wilson, A. Awonusi, M. D. Morris, D. H. Kohn, M. M. Tecklenburg, and L. W. Beck, Journal of Bone and Mineral Research 20, 625 (2004), ISSN 08840431, URL http://doi.wiley.com/10.1359/JBMR.041217.

[14] I. Brovchenko and A. Oleinikova, Interfacial and Confined Water (Elsevier, 2008), ISBN 978-0-444-52718-9.

[15] M.-C. Bellissent-Funel, S. H. Chen, and J.-M. Zanotti, Physical Review E 51, 4558 (1995), URL https://link.aps.org/doi/10.1103/PhysRevE. 51.4558

[16] D. F. Parsons, M. Bostrom, P. L. Nostro, and B. W. Ninham, Physical Chemistry Chemical Physics 13, 12352 (2011), ISSN 14639084, URL http://pubs.rsc.org/en/content/articlelanding/2011/ cp/c1cp20538b.

[17] V. Sansalone, J. Kaiser, S. Naili, and T. Lemaire, Biomechanics and Modeling in Mechanobiology 12, 533 (2013), ISSN 1617-7959, 1617-7940, URL https://link.springer.com/article/10.1007/s10237-012-0422-7.

[18] D. R. Wheeler and J. Newman, The Journal of Physical Chemistry B 108, 18353 (2004), ISSN 1520-6106, URL https://doi.org/10.1021/ jp047850b.

475 [19] E. J. Maginn, Accounts of Chemical Research (2007), URL http://pubs. acs.org/doi/full/10.1021/ar700163c.

[20] T. T. Pham, T. Lemaire, E. Capiez-Lernout, M. Lewerenz, Q.-D. To, J. K. Christie, D. D. Tommaso, N. H. Leeuw, and S. Naili, Theoretical 
Chemistry Accounts 134, 59 (2015), ISSN 1432-881X, 1432-2234, URL https://link.springer.com/article/10.1007/s00214-015-1653-3.

[21] M. Prakash, T. Lemaire, M. Caruel, M. Lewerenz, N. H. Leeuw, D. D. Tommaso, and S. Naili, Physics and Chemistry of Minerals 44, 509 (2017),

[23] D. Di Tommaso, M. Prakash, T. Lemaire, M. Lewerenz, N. H. de Leeuw, 490

[26] E. M. Calvo-Muoz, M. E. Selvan, R. Xiong, M. Ojha, D. J. Keffer, D. M. Nicholson, and T. Egami, Physical Review E 83, 011120 (2011), URL https://link.aps.org/doi/10.1103/PhysRevE.83.011120.

[27] H. Pan, J. Tao, T. Wu, and R. Tang, Frontiers of Chemistry in China 2, 156 (2007), ISSN 1673-3495, 1673-3614, URL https://link.springer. com/article/10.1007/s11458-007-0032-6

[28] P. Simonnin, B. Noetinger, C. Nieto-Draghi, V. Marry, and B. Rotenberg, 505 Journal of Chemical Theory and Computation 13, 2881 (2017), ISSN 15499618, URL https://doi.org/10.1021/acs . jctc.7b00342. 
[29] M. Vogele and G. Hummer, The Journal of Physical Chemistry B 120, 8722 (2016), ISSN 1520-6106, URL https://doi.org/10.1021/acs. jpcb.6b05102.

[30] K. Sudarsanan and R. A. Young, Acta Crystallographica Section B: Structural Crystallography and Crystal Chemistry 25, 1534 (1969), ISSN 05677408, URL http://scripts.iucr.org/cgi-bin/paper?a06841.

[31] D. Mkhonto and N. H. d. Leeuw, Journal of Materials Chemistry 12, 2633 (2002), ISSN 1364-5501, URL http://pubs.rsc.org/en/content/ articlelanding/2002/jm/b204111a

[32] N. H. de Leeuw, Physical Chemistry Chemical Physics 6, 1860

1 (2004), URL http://pubs.rsc.org/en/Content/ArticleLanding/2004/ CP/B313242K

[33] N. H. de Leeuw and S. C. Parker, Physical Review B 58, 13901 (1998), URL https://link.aps.org/doi/10.1103/PhysRevB.58.13901.

[34] H. J. C. Berendsen, J. R. Grigera, and T. P. Straatsma, The Journal of 口 Physical Chemistry 91, 6269 (1987), ISSN 0022-3654, URL http://dx. doi.org/10.1021/j100308a038.

[35] J.-F. Dufreche, O. Bernard, S. Durand-Vidal, and P. Turq, The Journal 525 of Physical Chemistry B 109, 9873 (2005), ISSN 1520-6106, URL https: //doi.org/10.1021/jp050387y.

[36] J. F. Dufreche, O. Bernard, and P. Turq, Journal of Molecular Liquids 118, 189 (2005), ISSN 0167-7322, URL http://www.sciencedirect.com/ science/article/pii/S0167732204001898.

[37] I. T. Todorov, W. Smith, K. Trachenko, and M. T. Dove, Journal of Mate$\square$ rials Chemistry 16, 1911 (2006), ISSN 1364-5501, URL http://pubs.rsc. org/en/content/articlelanding/2006/jm/b517931a. 
[38] T. Honorio, L. Brochard, and M. Vandamme, Langmuir 33, 12766 (2017),

п ISSN 0743-7463, URL http://dx.doi.org/10.1021/acs.langmuir. 7b03198.

[39] H. Scher and E. W. Montroll, Physical Review B 12, 2455 (1975), URL https://link.aps.org/doi/10.1103/PhysRevB.12.2455

[40] E. W. Montroll, Journal of Mathematical Physics 10, 753 (1969),

1. ISSN 0022-2488, URL https://aip.scitation.org/doi/abs/10.1063/ 1.1664902 .

[41] D. J. Keffer, B. J. Edwards, and P. Adhangale, Journal of Non-Newtonian Fluid Mechanics 120, 41 (2004), ISSN 0377-0257, URL http://www. sciencedirect.com/science/article/pii/S0377025704001053

[42] D. J. Keffer, C. Y. Gao, and B. J. Edwards, The Journal of Physical Chem545 istry B 109, 5279 (2005), ISSN 1520-6106, URL https://doi.org/10. 1021/jp0446635.

[43] A. Striolo, Nano Letters 6, 633 (2006), ISSN 1530-6984, URL http://dx. doi.org/10.1021/nl052254u.

[44] J. W. Swan and J. F. Brady, Physics of Fluids 22, 103301 (2010), ISSN 1070-6631, URL https://aip.scitation.org/doi/10.1063/1.3487748

[45] J. R. Blake and A. T. Chwang, Journal of Engineering Mathematics 8,

ㅁ 23 (1974), ISSN 0022-0833, 1573-2703, URL http://link.springer.com/ $10.1007 / \mathrm{BF} 02353701$

[46] L. Bocquet and J.-L. Barrat, EPL (Europhysics Letters) 31, 455 (1995), 555 I ISSN 0295-5075, URL http://stacks.iop.org/0295-5075/31/i=8/a= 006.

[47] R. M. Tinnacher, M. Holmboe, C. Tournassat, I. C. Bourg, and J. A. Davis, Geochimica et Cosmochimica Acta 177, 130 (2016), ISSN 
0016-7037, URL http://www.sciencedirect.com/science/article/ pii/S0016703715007048.

[48] I.-C. Yeh and G. Hummer, Biophysical Journal 86, 681 (2004), ISSN 0006-3495, URL http://www.sciencedirect.com/science/article/ pii/S0006349504741478.

[49] I.-C. Yeh and G. Hummer, The Journal of Physical Chemistry B 108, 15873 (2004), ISSN 1520-6106, URL http://dx.doi.org/10.1021/jp0477147.

[50] R. E. Zeebe, Geochimica et Cosmochimica Acta 75, 2483 (2011), ISSN 0016-7037, URL http://www.sciencedirect.com/science/article/ pii/S0016703711000895.

[51] A. J. Asta, M. Levesque, R. Vuilleumier, and B. Rotenberg, Physical Re570 view E 95, 061301 (2017), URL https://link.aps.org/doi/10.1103/ PhysRevE.95.061301

[52] J. S. Medina, R. Prosmiti, P. Villarreal, G. Delgado-Barrio, G. Winter, B. Gonzlez, J. V. Alemn, and C. Collado, Chemical Physics 388, 9 (2011), ISSN 0301-0104, URL/http://www.sciencedirect.com/science/ article/pii/S0301010411002813

[53] M. Holmboe and I. C. Bourg, The Journal of Physical Chemistry C 118, 1001 (2014), ISSN 1932-7447, URL http://dx.doi.org/10.1021/ jp408884g.

[54] B. Rotenberg, V. Marry, R. Vuilleumier, N. Malikova, C. Simon, and P. Turq, Geochimica et Cosmochimica Acta 71, 5089

a (2007), ISSN 0016-7037, URL http://www.sciencedirect.com/science/ article/pii/S0016703707005029

[55] S. Burov, R. Metzler, and E. Barkai, Proceedings of the National Academy 口 of Sciences 107, 13228 (2010), ISSN 0027-8424, 1091-6490, URL http: //www.pnas.org/content/107/30/13228 
[56] A. Kusumi, Y. Sako, and M. Yamamoto, Biophysical Journal 65, 2021

1 (1993), ISSN 0006-3495, URL http://wWw.sciencedirect.com/science/ article/pii/S0006349593812530.

[57] E. Kepten, I. Bronshtein, and Y. Garini, Physical Review E 87, 052713 (2013), URL https://link.aps.org/doi/10.1103/PhysRevE. 87.052713 .

[58] K. Burnecki, E. Kepten, Y. Garini, G. Sikora, and A. Weron, Scientific Reports 5, 11306 (2015), ISSN 2045-2322, URL https://www.nature.com/ articles/srep11306

[59] D. S. Martin, M. B. Forstner, and J. A. Kas, Biophysical Journal 83, 2109

口 (2002), ISSN 0006-3495, URL http://www.sciencedirect.com/science/ article/pii/S0006349502739714

[60] H. Qian, M. P. Sheetz, and E. L. Elson, Biophysical Journal 60, 910 (1991), ISSN 0006-3495, URL http://www.sciencedirect.com/science/ article/pii/S0006349591821257.

[61] E. Kepten, A. Weron, G. Sikora, K. Burnecki, and Y. Garini, PLoS ONE 10 (2015), ISSN 1932-6203, URL https://www.ncbi.nlm.nih.gov/pmc/ articles/PMC4334513/.

[62] S. Romero-Vargas Castrilln, N. Giovambattista, I. A. Aksay, and P. G. Debenedetti, The Journal of Physical Chemistry B 113, 7973 (2009), ISSN 1520-6106, URL https://doi.org/10.1021/jp9025392.

[63] Y. Marcus, Chemical Reviews 109, 1346 (2009), ISSN 0009-2665, URL http://dx.doi.org/10.1021/cr8003828. 